\title{
SCATTERING THEORY OF THE JOHNSON SPIN TRANSISTOR
}

\author{
L.S. Geux, A. Brataas and G.E.W. Bauer \\ Delft University of Technology, Laboratory of Applied Physics and DIMES \\ 2628 CJ Delft, The Netherlands
}

We discuss a simple, semiclassical scattering theory for spin-dependent transport in a many-terminal formulation, with special attention to the four terminal device of Johnson referred to as spin transistor.

PACS numbers: 73.40.Gk, 73.23.Hk, 75.70.Pa

\section{Introduction}

Transport in ferromagnets and ferromagnetic multilayers has become a popular subject of research since the discovery of the giant magnetoresistance (GMR) effect, but has been studied long before. Tedrow and Meservey [1] showed that the tunneling current in superconductor/insulator/ferromagnet junctions is spin dependent. Under an applied bias, the current of spin-up and spin-down electrons injected from a ferromagnet into a normal metal is different. This causes a non-equilibrium magnetization or "spin accumulation" which gives rise to an additional boundary resistance, the "spin-coupled interface resistance" [2, 3]. Johnson and Silsbee [2] detected this spin accumulation by two ferromagnetic contacts to a normal metal. Spin injection can be the physical basis of new devices, like the so-called spin transistor $[4,5]$. A "pedagogical model" of this device is shown in Fig. 1a. It consists of a normal metal film sandwiched between two ferromagnets. The magnetizations of the two ferromagnets are aligned either parallel or antiparallel. A spin-polarized current is injected from the first ferromagnet $F 1$ to a drain contact $P$ connected to the normal metal. The second ferromagnet $F 2$ is attached to a floating voltage probe. The polarized current creates a non-equilibrium magnetization in the normal metal, which splits the chemical potentials of the spin-up and spin-down electrons. Just as $F 1$ can be seen as a spin polarizer, $F 2$ can be seen as a spin detector, at which the chemical potential aligns with that of the spin-up (spin-down) electrons in the normal metal in the parallel (antiparallel) configuration. The voltage measured at $F 2$ is used to determine the impedance difference between the parallel and the antiparallel configuration, which is related to the spin-coupled resistance. The experiments are actually carried out with an additional normal metal counter electrode as in Fig. 1b. Spin-dependent transport 

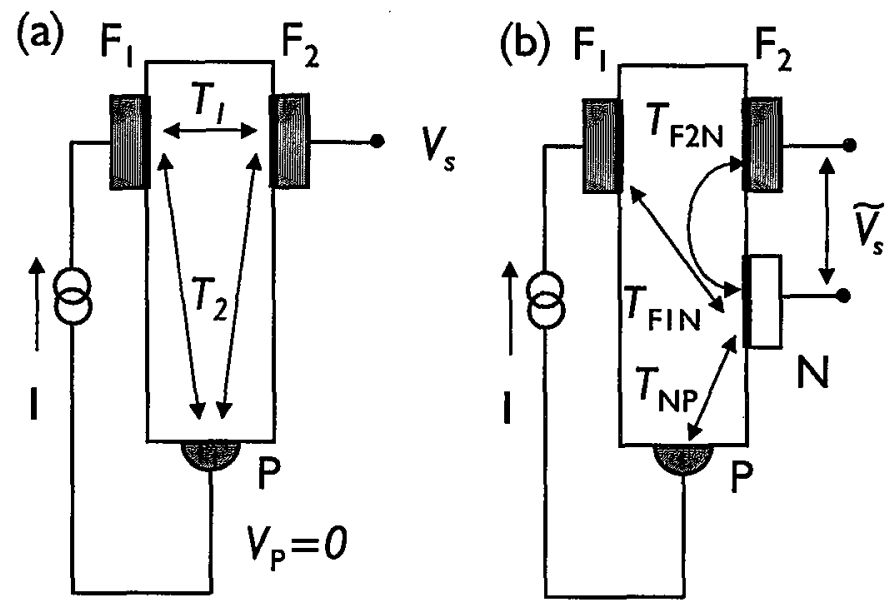

Fig. 1. Schematic picture of the spin transistor for (a) the pedagogical model and (b) the actual configuration.

is affected by the properties of the normal metal. Spin-flip scattering in the normal metal mixes the two spin channels and thus decreases the spin accumulation. The spin-orbit interaction [6] appears to limit the performance of the spin transistor. We discuss in this paper the multiterminal Landauer-Büttiker scattering theory of transport for a magnetic system. This formalism provides an alternative view on the physics of the device $[7,8]$ which in the diffusive regime is equivalent to previous two-terminal theories by Johnson and Fert and Lee [9], but we demonstrate that it can be extended to other regimes as well. For technical details we refer to [10].

\section{The spin transistor in the Landauer-Büttiker formalism}

In a two-terminal configuration the current from contact $A$ to contact $B$ is $I=G\left(\mu_{A}-\mu_{B}\right) e$, where $\Delta \mu=\mu_{A}-\mu_{B}$ is the difference in chemical potential between the two contacts. The linear response conductance $G$ is related to the transmission probability by the Landauer formula [7]:

$$
G=\frac{e^{2}}{h} \sum_{n m, \sigma \sigma^{\prime}}\left|t_{m \sigma, n \sigma^{\prime}}\right|^{2}
$$

where $t_{m \sigma, n \sigma^{\prime}}$ is the transmission amplitude of an electron from mode $m$ with spin $\sigma$ in lead $A$ to mode $n$ and spin $\sigma^{\prime}$ in lead $B$, all states being at the Fermi energy. Here we assume for simplicity that the magnetizations are collinear, i.e. parallel or antiparallel to each other. A network theory of magnetic multiterminal devices with general magnetization is presented in [11].

Büttiker extended the two-terminal Landauer formula to a many-terminal device by summing over all contacts [7]. If $T_{A \rightarrow B}$ is the total transmission probability from contact $A$ to $B$ :

$$
T_{A \rightarrow B}=\sum_{\sigma \sigma^{\prime}} \sum_{n}^{N_{B}} \sum_{m}^{N_{A}}\left|t_{m \sigma, n \sigma^{\prime}}\right|^{2},
$$


where $N_{A}$ is the number of modes in contact $A$ and $N_{B}$ - the number of modes in contact $B$. The total current in contact $A$ is given by

$$
I_{A}=\frac{e^{2}}{h}\left[\sum_{B(B \neq A)} T_{A \rightarrow B} \mu_{A}-\sum_{B(B \neq A)} T_{B \rightarrow A} \mu_{B}\right] .
$$

Referring to Fig. 1a, we call the transmission probability from $F 1$ to $F 2 T_{1}$. The transmission probability from both ferromagnetic contacts $F 1$ and $F 2$ to the drain contact is $T_{2}$. When the net current into $F 2$ is zero, the potential of $F 2$ can be obtained using Kirchhoff's law

$$
V_{s}=V \frac{T_{1}}{T_{1}+T_{2}}
$$

$V_{s}$ depends on the choice of the potential zero, here taken to be in the drain contact reservoir. On the other hand, $Z_{s}$, the impedance between $F 1$ and $P$, only depends on the potential difference $V$. A constant current $I$ is driven from $F 1$ into the drain contact of the paramagnetic film, which according to Eq. (3) is equal to

$$
I=-\frac{e^{2}}{h} T_{2}\left(V+V_{s}\right)
$$

The impedance $Z_{s}$ between $F 1$ and $P$ is therefore

$$
Z_{s}=\frac{V}{I}=-\frac{h}{e^{2}} \frac{1}{T_{2}} \frac{T_{1}+T_{2}}{2 T_{1}+T_{2}}=-\frac{h}{2 e^{2}}\left(\frac{1}{T_{2}}+\frac{1}{2 T_{1}+T_{2}}\right) .
$$

For the (pedagogical) system with two fully polarized ferromagnets without spin-flip processes the transmission probability between the two ferromagnets is zero when they are aligned antiparallel. The impedance $Z_{s}^{\mathrm{AP}}$ is then simply the reciprocal conductance between $F 1$ and $P$

$$
Z_{s}^{\mathrm{AP}}=-\frac{h}{e^{2}} \frac{1}{T_{2}} .
$$

The difference between the parallel and antiparallel configuration is

$$
Z_{s}^{\mathrm{P}}-Z_{s}^{\mathrm{AP}}=-Z_{s}^{\mathrm{AP}} \frac{T_{1} \mathrm{P}}{2 T_{1}^{\mathrm{P}}+T_{2}}
$$

where $T_{1}^{\mathrm{P}}$ is the transmission probability to $F 2$ in the parallel configuration.

In the presence of spin-flip scattering the transmission probability $T_{1}^{\mathrm{AP}}$ no longer vanishes. The difference in impedance between the parallel and antiparallel configuration now becomes

$$
Z_{s}^{\mathrm{P}}-Z_{s}^{\mathrm{AP}}=\frac{h}{e^{2}} \frac{T_{1}^{\mathrm{P}}-T_{1}^{\mathrm{AP}}}{4 T_{1}^{\mathrm{P}} T_{1}^{\mathrm{AP}}+2 T_{2}\left(T_{1}^{\mathrm{P}}+T_{1}^{\mathrm{AP}}\right)+T_{2}^{2}} .
$$

Johnson [4] implicitly assumed that the current into the drain contact did not affect the impedance difference. This can be modelled by an infinite resistance to the drain or $T_{2}=0$ :

$$
Z_{s}^{\mathrm{P}}-Z_{s}^{\mathrm{AP}}=\frac{h}{4 e^{2}}\left(\frac{1}{T_{1}^{\mathrm{AP}}}-\frac{1}{T_{1}^{\mathrm{P}}}\right)
$$

Equation (10) is equivalent to Johnson's result, which diverges in the absence of spin-flip scattering $T_{1}^{\mathrm{AP}} \rightarrow 0$. 
Instead of the ill-defined $V_{s}$, Johnson measured a voltage $\tilde{V}_{s}$ with respect to a normal counter electrode $N$, cf. Fig. 1b. The transmission probabilities from $F 1$ and $F 2$ to the normal counterelectrode $N$ are called $T_{F 1 N}$ and $T_{F 2 N}$, respectively and $T_{N P}$ is the transmission probability from $N$ to $P$. Again setting the net current into $F 2$ and $N$ to zero

$$
Z_{s}=\frac{\widetilde{V}_{s}}{I}=\frac{h}{e^{2}} \frac{1}{D}\left(T_{1} T_{N P}-T_{F 1 N} T_{2}\right)
$$

where

$$
\begin{aligned}
D= & T_{2}^{2} T_{N P}+T_{2}\left(T_{2}+T_{N P}\right)\left(T_{F 1 N}+T_{F 2 N}\right) \\
& +2 T_{2} T_{N P} T_{1}+\left(2 T_{2}+T_{N P}\right)\left(T_{1} T_{F 1 N}+T_{1} T_{F 2 N}+T_{F 1 N} T_{F 2 N}\right) .
\end{aligned}
$$

Assuming that the resistance to the drain contact is relatively large, we can consider two limits. When the normal counterelectrode $N$ is positioned far from the drain contact $P$ both transmission probabilities to the drain contact, $T_{2}$ and $T_{N P}$, vanish and the impedance of the system reduces to

$$
Z_{s}=\frac{\tilde{V}_{s}}{I}=\frac{h}{3 e^{2}} \frac{T_{1}-T_{F 1 N}}{T_{1}\left(T_{F 1 N}+T_{F 2 N}\right)+T_{F 1 N} T_{F 2 N}} .
$$

The impedance difference is

$$
\begin{aligned}
Z_{s}^{\mathrm{P}} & -Z_{s}^{\mathrm{AP}}=\frac{h}{3 e^{2}} \frac{\left(T_{1}^{\mathrm{P}}-T_{1}^{\mathrm{AP}}\right) T_{F 1 N}\left(T_{F 1 N}+2 T_{F 2 N}\right)}{\left[T_{1}^{\mathrm{P}}\left(T_{F 1 N}+T_{F 2 N}\right)+T_{F 1 N} T_{F 2 N}\right]} \\
& \times \frac{1}{\left[T_{1}^{\mathrm{AP}}\left(T_{F 1 N}+T_{F 2 N}\right)+T_{F 1 N} T_{F 2 N}\right]}
\end{aligned}
$$

Comparing Eq. (14) with Eq. (10), we expect the four-terminal configuration to be equivalent to the three-terminal configuration when $T_{F 2 N} \ll T_{F 1 N}$. However, the impedance difference has a maximum for $T_{F 2 N}=0$ :

$$
Z_{s}^{\mathrm{P}}-Z_{s}^{\mathrm{AP}}=\frac{h}{3 e^{2}}\left(\frac{1}{T_{1}^{\mathrm{AP}}}-\frac{1}{T_{1}^{\mathrm{P}}}\right),
$$

which is increased by a factor $4 / 3$ compared to the pedagogical model.

When the normal counterelectrode $N$ is positioned close to the drain contact $P, T_{N P}$ is no longer negligible and $T_{F 1 N}$ and $T_{F 2 N}$ are small. The impedance difference

$$
Z_{s}^{\mathrm{P}}-Z_{s}^{\mathrm{AP}}=\frac{h}{e^{2}}\left(\frac{1}{T_{1}^{\mathrm{AP}}}-\frac{1}{T_{1}^{\mathrm{P}}}\right) \frac{T_{F 1 N} T_{F 2 N}}{\left(T_{F 1 N}+T_{F 2 N}\right)^{2}}
$$

now depends on the ratio of $T_{F 1 N}$ and $T_{F 2 N}$. It has a maximum for $T_{F 1 N}=T_{F 2 N}$ for which it reduces to Eq. (10). Apparently, the impedance difference is affected by the choice of position of the normal counterelectrode $N$.

A microscopic calculation of the various transmission probabilities involves bulk, spin-dependent interface and spin-flip scattering processes. We present here a first attempt based on a semiclassical calculation of a weakly scattering slice of a disordered material. 


\section{Sheet impurity scattering with spin flip}

In the effective mass approximation the single-electron states at the Fermi energy $E_{\mathrm{F}}$ are described by the Schrödinger equation in a normal metal

$$
\left[\left(-\frac{\hbar^{2}}{2 m^{*}} \nabla^{2}+V(r)-E_{\mathrm{F}}\right) \mathcal{I}+\mathcal{H}^{\mathrm{sg}}(r)+\mathcal{H}^{\mathrm{so}}(r)\right]\left(\begin{array}{c}
\psi_{\uparrow}(r) \\
\psi_{\downarrow}(r)
\end{array}\right)=0
$$

where $m^{*}$ is the effective mass. We consider here the effect of a thin sheet of short-range impurities with a scalar scattering potential $V(r)=\sum_{\alpha} \gamma_{\alpha} \delta(z)$ $\times \delta\left(\rho-\rho_{\alpha}\right)$, where $\rho_{\alpha}$ gives the transverse position of the scattering center, and $\gamma_{\alpha}$ gives the strength of the scatterer. Spin-flip scattering can be induced by spin-orbit scatterers [12]

$$
\left.\mathcal{H}^{\text {so }}=\frac{\hbar}{4 m^{2} c^{2}} \boldsymbol{\sigma} \cdot[(\nabla V(r)) \times(-\mathrm{i} \hbar \nabla))\right],
$$

where $\sigma$ is the vector containing the Pauli spin matrices, or interaction with magnetic impurities with fixed random spin direction (spin glass)

$$
\mathcal{H}^{\mathrm{sg}}=\sum_{\alpha} J_{\mathrm{ex}} \boldsymbol{S}_{\alpha} \cdot \boldsymbol{\sigma} \delta(z) \delta\left(\rho-\rho_{\alpha}\right)
$$

Here $\boldsymbol{S}_{\alpha}$ is the spin of the paramagnetic impurity and $J_{\mathrm{ex}}$ is the local exchange integral.

We wish to compute the Green function $[13,14,8]$

$$
\begin{aligned}
& G^{ \pm}\left(\boldsymbol{r}, \sigma ; \boldsymbol{r}^{\prime}, \sigma^{\prime}\right) \\
& \quad=\sum_{\boldsymbol{k}_{\|}, \boldsymbol{k}_{\|}^{\prime}} G_{k_{\|} \sigma, k_{\|}^{\prime} \sigma^{\prime}}^{+} \exp \left(\mathrm{i} k_{\perp} z_{\mathrm{R}}\right) \exp \left(\mathrm{i} k_{\|} \rho_{\mathrm{R}}\right) \exp \left(-\mathrm{i} k_{\perp}^{\prime} z_{\mathrm{L}}\right) \exp \left(-\mathrm{i} k_{\|}^{\prime} \rho_{\mathrm{L}}\right),
\end{aligned}
$$

where $r_{\mathrm{R}, \mathrm{L}}=\left(\rho_{\mathrm{R}, \mathrm{L}} \mathrm{em}, \mathrm{z}_{\mathrm{R}, \mathrm{L}}\right)$ and $z_{\mathrm{R}}>0, z_{\mathrm{L}}<0$ are located in the right and left leads. Without perturbations

$$
G_{\boldsymbol{k}_{\|} \sigma, \boldsymbol{k}_{\|}^{\prime} \sigma^{\prime}}^{+} \rightarrow G_{\boldsymbol{k}_{\|}}^{+(0)} \delta_{\boldsymbol{k}_{\|}, \boldsymbol{k}_{\|}^{\prime}} \delta_{\sigma, \sigma^{\prime}}=-\mathrm{i} \frac{m^{*}}{\hbar^{2}} \frac{1}{k_{\perp}} \delta_{\boldsymbol{k}_{\|}, \boldsymbol{k}_{\|}^{\prime}} \delta_{\sigma, \sigma^{\prime}}
$$

The matrix of transmission coefficients is related to the Green function by [13]

$$
t_{\boldsymbol{k}_{\|} \sigma, \boldsymbol{k}_{\|}^{\prime} \sigma^{\prime}}=\frac{\mathrm{i} \hbar^{2}}{m} \sqrt{\left|k_{\perp} \| k_{\perp}^{\prime}\right|} G_{\boldsymbol{k}_{\|} \sigma, \boldsymbol{k}_{\|}^{\prime} \sigma^{\prime}}^{+}
$$

For isolated interfaces the following optical theorem holds [14]:

$$
\sum_{\sigma} \sum_{\boldsymbol{k}_{\|}}^{\left|\boldsymbol{k}_{\|}\right| \leq k_{\mathrm{F}}}\left|G_{k_{\|} \sigma, k_{\|}^{\prime} \sigma^{\prime}}^{+}\right|^{2} / G_{k_{\|}}^{+(0)}=-\mathrm{i} \operatorname{Im}\left(G_{k_{\|}^{\prime} \sigma^{\prime}, \boldsymbol{k}_{\|}^{\prime} \sigma^{\prime}}^{+}\right) \text {. }
$$

We are interested in the transport properties averaged over impurity configurations. The averaged Green function reads

$$
\left\langle G_{\boldsymbol{k}_{\|} \sigma, \boldsymbol{k}_{\|}^{\prime} \sigma^{\prime}}^{+}\right\rangle=\left(G_{\boldsymbol{k}_{\|}}^{+(0)-1}-\Sigma_{\boldsymbol{k}_{\|}}\right)^{-1} \delta_{\boldsymbol{k}_{\|}, \boldsymbol{k}_{\|}^{\prime}} \delta_{\sigma, \sigma^{\prime}} .
$$


The conductance can be calculated in two ways. By using Eq. (23) and the relation (22) between the Green function and the transmission coefficients

$$
G=\frac{2 e^{2}}{h} \sum_{\boldsymbol{k}_{\|}}^{\left|\boldsymbol{k}_{\|}\right| \leq k_{\mathrm{F}}} \frac{1-\left(G_{k_{\|}}^{+(0)}\right) \mathrm{i} \operatorname{Im} \Sigma_{\boldsymbol{k}_{\|}}}{\left|1-G_{\boldsymbol{k}_{\|}}^{+(0)} \Sigma_{\boldsymbol{k}_{\|}}\right|^{2}} .
$$

Alternatively, the conductance can be calculated diagrammatically via the transmission probabilities, which can be written in general as

$$
\begin{aligned}
& \left\langle\left|t_{\boldsymbol{k}_{\|} \sigma, k_{\|}^{\prime} \sigma^{\prime}}\right|^{2}\right\rangle=\frac{1}{\mid 1-G_{\boldsymbol{k}_{\|}}^{+(0)} \Sigma_{\left.\boldsymbol{k}_{\|}\right|^{2}}} \delta_{\boldsymbol{k}_{\|}, \boldsymbol{k}_{\|}^{\prime}} \delta_{\sigma, \sigma^{\prime}} \\
& +\frac{G_{\boldsymbol{k}_{\|}}^{+(0) *}}{\left|1-G_{\boldsymbol{k}_{\|}}^{+(0)} \Sigma_{\left.\boldsymbol{k}_{\|}\right|^{2}}\right|^{2}} W_{\boldsymbol{k}_{\|} \sigma, k_{\|}^{\prime} \sigma^{\prime}} \frac{G_{\boldsymbol{k}_{\|}^{\prime}}^{+(0)}}{\left|1-G_{\boldsymbol{k}_{\|}^{\prime}}^{+(0)} \Sigma_{\left.\boldsymbol{k}_{\|}\right|^{2}}\right|^{2}} .
\end{aligned}
$$

Diffuse scattering and spin diffusion are described by the second term of Eq. (26) which is governed by the (reducible) vertex correction $W$.

These formally exact relations can be evaluated for the Born approximation which is valid for low impurity densities $n_{I R}=N_{I R} / A$ and weak scattering strength. With an average strength of the scatterers $\bar{\gamma}=\sum_{\alpha} \gamma_{\alpha, \sigma} / N_{I R}$ and a mean square value $\gamma^{2}=\sum_{\alpha} \gamma_{\alpha, \sigma}^{2} / N_{I R}$, the self-energy reads in the Born approximation

$$
\Sigma^{\mathrm{B}}=n_{I R} \bar{\gamma}-\mathrm{i} \frac{\hbar^{2}}{m^{*}} k_{\mathrm{F}}\left(\eta+\eta_{\mathrm{sf}}\right)
$$

where $\eta$ is the spin-conserving scattering parameter

$$
\eta=\frac{n_{I R}}{2 \pi}\left(\frac{m^{*} \gamma}{\hbar^{2}}\right)^{2}
$$

$\eta_{\mathrm{sf}}=\eta_{\mathrm{sf}}^{\mathrm{so}}+\eta_{\mathrm{sf}}^{\mathrm{sg}}$ is the spin-flip scattering parameter caused by spin-orbit scatterers with $u_{\mathrm{so}}=\hbar^{2} k_{\mathrm{F}}^{2} / 4 m^{2} c^{2}$ :

$$
\eta_{\mathrm{sf}}^{\mathrm{so}}=\frac{2}{3} \frac{n_{I R}}{2 \pi}\left(\frac{m^{*} u_{\mathrm{so}} \gamma}{\hbar^{2}}\right)^{2}
$$

and by paramagnetic impurities

$$
\eta_{\mathrm{sf}}^{\mathrm{sg}}=\frac{n_{I R}}{2 \pi}\left(\frac{m^{*} J}{\hbar^{2}}\right)^{2}
$$

Assuming $\operatorname{Re} \Sigma^{\mathrm{B}} \ll \operatorname{Im} \Sigma^{\mathrm{B}}$ and inserting Eq. (21) into Eq. (25)

$$
G=\frac{2 e^{2}}{h} \sum_{\boldsymbol{k}_{\|}}^{\left|k_{\|}\right| \leq k_{\mathrm{F}}} \frac{k_{\perp}}{k_{\perp}+\left(\eta+\eta_{\mathrm{sf}}\right) k_{\mathrm{F}}} .
$$

To the first order in $\eta$ the conductance is given by $G / G_{0}=1-2\left(\eta+\eta_{\mathrm{sf}}\right)$, where $G_{0}=\left(2 e^{2} / h\right)\left(A k_{\mathrm{F}}^{2} / 4 \pi\right)$ is the Sharvin conductance. In order to calculate the spin 
diffusion, we need the transmission probability for spin-flip scattering. To the lowest order in the scattering parameters the transmission probability matrix in spin space is obtained by summing $\left\langle\left|t_{k_{\|}, \boldsymbol{k}_{\|}}\right|^{2}\right\rangle$ over the incoming and outgoing states. The spin-flip probability $T_{\mathrm{sf}}$ under transmission is

$$
T_{\mathrm{sf}}=\frac{A k_{\mathrm{F}}^{2}}{2 \pi} \frac{2}{3} \eta_{\mathrm{sf}}
$$

whereas the spin-conserving transmission probability $T$ is

$$
T=\frac{A k_{\mathrm{F}}^{2}}{2 \pi}\left[\frac{1}{2}-\left(\eta+\frac{5}{3} \eta_{\mathrm{sf}}\right)\right] \text {. }
$$

\section{Finite thickness}

\subsection{Relevant length scales}

Next to the geometrical parameters of a sample, i.e. the sample cross-sections $A$ and length $L$, several characteristic length scales govern the transport properties. In the (quasi-) ballistic regime the conductance is dominated by the contact resistance. In the diffuse regime transport is limited by scattering at bulk impurities. An obvious parameter is the impurity scattering mean free path $\ell$. The spin-fip mean free path $\ell_{\mathrm{sf}}$ is the average length that an electron travels before it flips its spin. Including scattering at spin-orbit scatterers or paramagnetic impurities, does not only decrease $\ell_{\mathrm{sf}}=v_{\mathrm{F}} \tau_{\mathrm{sf}}$, where $\tau_{\mathrm{sf}}$ is the spin-flip scattering time and $v_{\mathrm{F}}$ the Fermi velocity, but also reduces the mean free path $\ell^{-1}=v_{\mathrm{F}}^{-1}\left(\tau^{-1}+\tau_{\mathrm{sf}}^{-1}\right)$. In the diffuse regime the relevant parameter is not $\ell_{\mathrm{sf}}$, but the spin diffusion length $l_{\mathrm{sf}}=\sqrt{\ell \ell_{\mathrm{sf}} / 6}$ over which the spin-accumulation persists [15].

\subsection{Two-terminal conductance}

The transport properties of samples with finite thickness can be readily obtained from the results of the previous chapter in two different limits. When the mean-free path is much larger than the current path, the system is in the quasiballistic limit and the results above can be carried over directly. In the quasiballistic regime, $L \ll \ell$, we can make the connection of the scattering mean free paths and the microscopic parameters $N\left(\eta+\eta_{\mathrm{sf}}\right)=L / 2 \ell$ and $N \eta_{\mathrm{sf}}=L / 2 \ell_{\mathrm{sf}}$. The conductance is $G^{q b}(L) / G_{0}=1-2 L / \ell$. The spin-flip probability for transmission through a thin slice of bulk material follows from Eq. (32):

$$
T_{\mathrm{sf}}^{q b}(L)=\frac{A k_{\mathrm{F}}^{2}}{2 \pi} \frac{2}{3} N \eta_{\mathrm{sf}}=\frac{A k_{\mathrm{F}}^{2}}{4 \pi} \frac{L}{\ell_{\mathrm{sf}}}
$$

and for transmission without spin flip

$$
T^{q b}(L)=\frac{A k_{\mathrm{F}}^{2}}{4 \pi}\left(1-\frac{L}{\ell}-\frac{L}{\ell_{\mathrm{sf}}}\right) .
$$

In the diffuse regime, where $L \gg \ell$ or $N\left(\eta+\eta_{\mathrm{sf}}\right) \gg 1$, the conductance is $G^{d f}(L) / G_{0}=4 \ell / 3 L$. The spin-resolved two-terminal transmission probabilities in the diffuse regime, on the other hand, can be obtained from the scattering properties of a thin slice by following Schep et al. [16]. It is shown in [10] that this 
procedure is equivalent to solving the diffusion equation $[15,9]$ with the following general solutions for the spin-average $\bar{\mu}=A z+B$ and the spin-splitting $\delta \mu$ of the chemical potentials

$$
\delta \mu(z)=C \exp \left(-z / l_{\mathrm{sf}}\right)+D \exp \left(z / l_{\mathrm{sf}}\right) .
$$

The integration constants $A, B, C, D$ have to be determined by the boundary conditions. The transport properties are easily obtained from the chemical potentials.

\section{Spin transistor}

We can now collect the different results to obtain expressions for the spin transistor. We approximate the transmission probabilities in the many-terminal configuration by those in the two-terminal configuration. This is not unproblematic because we do not know the current paths. Complication are for example spreading resistances at contacts, which reflect the fact that electrons do not always travel straight between the two contacts. However, in a thin film geometry like the spin transistor, we expect the corrections to be not too large for a qualitative study. It should be kept in mind that the geometric parameters, i.e. the cross-section $A$ and the current path length $L$, may somewhat deviate from the geometrical measures of the sample.

\subsection{Quasiballistic regime}

Let us consider first the quasiballistic regime and assume that the contacts are made from strong ferromagnets in which the minority carrier density vanishes and the majority density equals that of the normal metal island. In this limit the performance of the spin-transistor is determined by the spin-flip scattering length $\ell_{\text {sf }}>\ell$. From Eqs. (34) and (35), the transmission probabilities are

$$
T_{1}^{\mathrm{P}}=\frac{A k_{\mathrm{F}}^{2}}{4 \pi}\left(1-\frac{L_{1}}{\ell}-\frac{L_{1}}{\ell_{\mathrm{sf}}}\right)
$$

and

$$
T_{1}^{\mathrm{AP}}=\frac{A k_{\mathrm{F}}^{2}}{4 \pi} \frac{L_{1}}{\ell_{\mathrm{sf}}} .
$$

The transmission probability to the drain contact can be either quasiballistic or ohmic, depending on the distance $L_{2}$ between the ferromagnetic and drain contact. If the drain channel is quasiballistic $\left(L_{2} \ll \ell\right)$

$$
T_{2}=\frac{A k_{\mathrm{F}}^{2}}{4 \pi}\left(1-\frac{L_{2}}{\ell}\right) \text {. }
$$

The impedance difference for the pedagogical model is to lowest order in $L_{1}$ :

$$
\left[Z_{s}^{\mathrm{P}}-Z_{s}^{\mathrm{AP}}\right]^{q b}=\frac{1}{G_{0}} \frac{3 L_{2}}{8 \ell}\left(1-\frac{3 L_{2}}{4 \ell} \frac{L_{1}}{\ell_{\mathrm{sf}}}\right) .
$$

This equation also holds when transport to the drain is diffusive. 


\subsection{Diffuse regime}

Let us now consider tunnel contacts with a spin-injection efficiency which can be expressed in terms of $G_{\sigma}^{\mathrm{F}}$, the tunneling conductance for spin $\sigma$, respectively the density of states at the Fermi energy $N_{\sigma}\left(E_{\mathrm{F}}\right)$ :

$$
\beta=\frac{N_{\uparrow}\left(E_{\mathrm{F}}\right)-N_{\downarrow}\left(E_{\mathrm{F}}\right)}{N_{\uparrow}\left(E_{\mathrm{F}}\right)+N_{\downarrow}\left(E_{\mathrm{F}}\right)}=\frac{G_{\uparrow}^{\mathrm{F}}-G_{\downarrow}^{\mathrm{F}}}{\mathrm{G}_{\uparrow}^{\mathrm{F}}+\mathrm{G}_{\downarrow}^{\mathrm{F}}} .
$$

We will now consider a system consisting of a normal metal of thickness $L_{N}$. sandwiched between two ferromagnetic tunneling junctions, over which a voltage $\Delta \mu / e$ is applied. Defining the average tunneling conductivity $G_{\mathrm{F}}=2 G_{\uparrow}^{\mathrm{F}} G_{\downarrow}^{\mathrm{F}} /$ $\left(G_{\uparrow}^{\mathrm{F}}+G_{\downarrow}^{\mathrm{F}}\right)$ we can solve the diffusion equations, with the result for the transmission coefficients

$$
\begin{aligned}
& \frac{1}{T_{1}^{\mathrm{P}}}=\frac{L_{1}}{4 g_{N}}+\frac{1}{2} \frac{\sinh \left(L_{N} / 2 l_{\mathrm{sf}}\right)+\left(1-\beta^{2}\right) \frac{g_{N}}{l_{\mathrm{sf}}} \frac{1}{G_{\mathrm{F}}} \cosh \left(L_{N} / 2 l_{\mathrm{sf}}\right)}{G_{\mathrm{F}} \sinh \left(L_{N} / 2 l_{\mathrm{sf}}\right)+g_{N} \cosh \left(L_{N} / 2 l_{\mathrm{sf}}\right) / l_{\mathrm{sf}}} \\
& \frac{1}{T_{1}^{\mathrm{AP}}}=\frac{L_{1}}{4 g_{N}}+\frac{1}{2} \frac{\cosh \left(L_{N} / 2 l_{\mathrm{sf}}\right)+\left(1-\beta^{2}\right) \frac{1}{G_{\mathrm{F}}} \frac{g_{N}}{l_{\mathrm{s}}} \sinh \left(L_{N} / 2 l_{\mathrm{sf}}\right)}{G_{\mathrm{F}} \cosh \left(L_{N} / 2 l_{\mathrm{sf}}\right)+g_{N} \sinh \left(L_{N} / 2 l_{\mathrm{sf}}\right) / l_{\mathrm{sf}}} \\
& \frac{1}{T_{\mathrm{F}} N}=\frac{L_{2}}{4 g_{N}}+\frac{1}{4} \frac{\sinh \left(L_{2} / l_{\mathrm{sf}}\right)+\left(1-\beta^{2}\right) \frac{1}{G_{\mathrm{F}}} \frac{g_{N}}{l_{\mathrm{sf}}} \cosh \left(L_{2} / l_{\mathrm{sf}}\right)}{G_{\mathrm{F}} \sinh \left(L_{2} / l_{\mathrm{sf}}\right)+g_{N} \cosh \left(L_{2} / l_{\mathrm{sf}}\right) / l_{\mathrm{sf}}}
\end{aligned}
$$

where $g_{N}=A k_{\mathrm{F}}^{2} \ell / 3 \pi$. In the limit $T_{2} \rightarrow 0$ the impedance difference becomes

$$
\begin{aligned}
Z_{s}^{\mathrm{P}} & -Z_{s}^{\mathrm{AP}} \\
& =\frac{2 \rho_{N}}{A} \frac{\beta^{2} l_{\mathrm{sf}}}{\left[\left(l_{\mathrm{sf}} G_{\mathrm{F}} / g_{N}\right)^{2}+1\right] \sinh \left(L_{1} / l_{\mathrm{sf}}\right)+2 G_{\mathrm{F}} l_{\mathrm{sf}} \cosh \left(L_{1} / l_{\mathrm{sf}}\right) / g_{N}}
\end{aligned}
$$

has a maximum for $G_{\mathrm{F}}=0$ :

$$
Z_{s}^{\mathrm{P}}-Z_{s}^{\mathrm{AP}}=\frac{2 \rho_{N}}{A} \frac{\beta^{2} l_{\mathrm{sf}}}{\sinh \left(L_{1} / l_{\mathrm{sf}}\right)}
$$

which agrees with [9] in the tunneling limit.

By an analysis of his experiments Johnson found $l_{\mathrm{sf}}=1.5 \pm 0.4 \mu \mathrm{m}$ [5]. The impedance of the spin transistor is approximately $25 \mu \Omega$ in the parallel configuration and the measured impedance is $3 \mu \Omega$ for the $1.6 \mu \mathrm{m}$ thick film. The largest impedance difference predicted for the optimum value $|\beta|=1$, using Eq. (46) and the above value found for $l_{\mathrm{sf}}$, is $Z_{s} A d \approx 3 \times 10^{-2} \Omega \mu \mathrm{m}^{3}$, which is about three times smaller than the measured difference. This problem might be caused by contamination which reduces the contact area.

\section{Conclusions}

We analyzed the spin transistor by a semiclassical scattering theory of transport resolving some inconsistencies which arise from a two-terminal approximation for a many-terminal device. The performance of the spin transistor is limited by 
the spin-diffusion length in the diffuse regime and the spin-flip scattering length in the quasiballistic regime. Naturally, we may expect a better transistor action in clean samples.

\section{Acknowledgments}

This work is part of the research program for the "Stichting voor Fundamenteel Onderzoek der Materie" (FOM), which is financially supported by the "Nederlandse Organisatie voor Wetenschappelijk Onderzoek" (NWO). This study was supported by the NEDO joint research program (NTDP-98). We acknowledge benefits from the TMR Research Network on "Interface Magnetism" under contract No. FMRX-CT96-0089 (DG12-MIHT). We also acknowledge stimulating discussions with K.M. Schep, J. Caro, P.J. Kelly, and Yu.V. Nazarov.

\section{References}

[1] P.M. Tedrow, R. Meservey, Phys. Rev. Lett. 26, 192 (1971).

[2] M. Johnson, R.H. Silsbee, Phys. Rev. B 37, 5321 (1988); M. Johnson, R.H. Silsbee, Phys. Rev. B 37, 5326 (1988).

[3] P.C. van Son, H. van Kempen, P. Wyder, Phys. Rev. Lett. 58, 2271 (1987).

[4] M. Johnson, J. Appl. Phys. 75, 6714 (1994).

[5] M. Johnson, Science 260, 320 (1993).

[6] Y. Yafet, in: Solid State Physics, Eds. F. Seitz, D. Turnbull, Vol. 14, Academic, New York 1963, p. 1.

[7] S. Datta, Electronic Transport in Mesoscopic Systems, Cambridge University Press, Cambridge 1995.

[8] M.A.M. Gijs, G.E.W. Bauer, Adv. Phys. 46, 285 (1997) and references therein.

[9] A. Fert, S. Lee, Phys. Rev. B 53, 6554 (1996).

[10] L.S. Geux, Diploma Thesis, Department of Applied Physics, TU Delft, 1997.

[11] A. Brataas, Yu.V. Nazarov, G.E.W. Bauer, preprint available on http://xxx.lanl.gov, cond-mat/9906065.

[12] L.I. Schiff, Quantum Mechanics, McGraw-Hill, Kogakusha 1955.

[13] D.S. Fisher, P.A. Lee, Phys. Rev. B 23, 6851 (1981).

[14] A. Brataas, G.E.W. Bauer, Phys. Rev. B 49, 14684 (1994).

[15] T. Valet, A. Fert, Phys. Rev. B 48, 7099 (1993).

[16] K.M. Schep, J.B.A.N. van Hoof, G.E.W. Bauer, J.E. Inglesfield, Phys. Rev. B 56, 10805 (1997). 